\title{
La calidad de los servicios médicos asistenciales: estudio de caso en una institución privada
}

\section{RESUMEN}

El objetivo del presente trabajo es evaluar la calidad de servicio percibida por los usuarios de un centro médico privado en Valencia, Venezuela. El instrumento para la recolección de datos se basó en las percepciones del SERVQUAL. Se identificaron, mediante el Análisis de Factores, una vez demostrada la pertinencia muestral, tres (03) dimensiones: Seguridad, Confiabilidad y Tangibilidad, que explican $66,63 \%$ de la varianza total; así mismo, el instrumento presentó consistencia interna (alfa de cronbach >0,955) por lo que resulta fiable y los resultados de las pruebas estadísticas realizadas (Significativas al $5 \%$ ), evidencian la validez del mismo. Con base en los resultados, se concluye que el cuestionario reúne suficientes condiciones para ser utilizado como medida de la calidad percibida por los pacientes del centro médico privado, y como insumo para el diseño de estrategias..

Palabras clave: calidad de servicio, universidad, análisis de factoalidad de servicio, Salud, SERVQUAL, Análisis de Factoresl

Medical Services Quality: A CaSe STUdy in PRIVATE INSTITUTION

\section{ABSTRACT}

The objective of this study is to evaluate the quality of service perceived by users in a private medical center in Valencia, Venezuela. The instrument for data collection was based on the perceptions of SERVQUAL. Were identified by Factor Analysis, once they demonstrated the relevance sample, three (03) dimensions: Safety, Reliability and tangibility, explaining $66.63 \%$ of the total variance, likewise, the instrument showed internal consistency (alpha cronbach $>0.955$ ) as it reliable and results of statistical tests performed (Significant at $5 \%$ ), demonstrate the validity. Based on the results, we conclude that the questionnaire has enough condition to be used as a measure of the quality perceived by patients private medical center, and as an input for the design of strategies.

Keywords: Service Quality, Health, SERVQUAL, Factor Analysis.

\section{INTRODUCCIÓN}

El carácter dinámico de las organizaciones inteligentes predominantes en esta segunda década del siglo XXI, ha hecho que los profesionales responsables de liderar los procesos de cambio, tengan que mantenerse en sintonía con las nuevas tendencias. Hoy en día, resulta esencial disponer de organizaciones competitivas, adaptables a los constantes cambios tecnológicos y capaces de responder a la cada vez más exigente demanda de los mercados y el sector sanitario no se queda atrás en este sentido (Díaz, 2005).

En lo que a calidad se refiere, ésta ha existido desde siempre, con diferentes niveles de desarrollo según las necesidades humanas. La primera demostración de calidad se evidencia en el libro Génesis (el primer libro de la Biblia) al narrar la creación del mundo en seis días, en el cual se describe como al final de cada día Dios veía al terminar su obra como ésta "era buena".

Existen diferentes demostraciones de calidad a través de la historia, pero la época en donde la calidad juega un papel más relevante es en el paso de la producción artesanal a la producción en serie (Revolución Industrial) porque desaparece la relación fabricante y cliente en presencia del producto ocasionando un aumento de productos defectuosos que salen de las cadenas de producción; surgiendo de esta manera el concepto de control estadístico de calidad en el cual la calidad se entiende como "conformidad con la especificación".

En la actualidad la Norma Internacional ISO 9000 (2005) plantea una definición de la calidad que aun cuando es genérica, permite enfocar las iniciativas en este sentido; es así como comúnmente se define la calidad como "el grado en el que un conjunto de características inherentes cumple con los requisitos".

Por su parte, el conocido estadístico estadounidense William Edwards Deming en su libro "Fuera de la crisis" (1988) menciona argumentos principales con respecto al concepto de calidad los cuales, vale la pena señalar:

- La calidad tiene que estar definida en términos de satisfacción del cliente.

\footnotetext{
Ingeniero Industrial, Docente Investigador Universidad de Valparaiso, Chile. E-mail: mleyzeaga23@gmail.com

** Ingeniero Industrial, Universidad de Sonora, México. E-mail: jaan_1991@hotmail.com

*** Ingeniero Industrial, M. Sc. Dr., Docente Investigador Universidad de Carabobo, Venezuela. E-mail: aamejiasa@gmail.com
} 
- La calidad es multidimensional. Es virtualmente imposible definir calidad de un producto o servicio en términos de una simple característica $o$ agente.

La calidad puede estar enfocada hacia un producto o hacia un servicio, siendo esta para un producto más tangible que para un servicio; Parasuraman et al. (1991) consideran que un cliente percibe un servicio como de alta calidad cuando su experiencia concreta con la empresa iguala o supera sus expectativas iniciales, razón por la cual surge un tema de gran relevancia en la actualidad como lo es la medición de la calidad del servicio.

Grandes estudiosos de la calidad de servicios como lo son Zeithalm, Berry y Parasuraman desarrollaron un modelo para medir y mejorar la calidad de servicio este modelo es conocido como SERVQUAL (1988, 1991). EI SERVQUAL es un modelo que se basa en cinco (5) dimensiones para lograr medir la calidad de servicio, las cuales son: fiabilidad, capacidad de respuesta, seguridad, empatía y elementos tangibles. Constituye una escala de respuestas múltiples para entender las expectativas de los clientes respecto a un servicio; busca mejorar la calidad de servicio en las empresas que lo implementen con éxito, logrando lo que toda gerencia desea como lo es alcanzar la fidelidad de los clientes.

El estudio reportado en este artículo, se enfoca hacia el sector de servicios de salud, específicamente en el privado y tiene como objetivo principal identificar las dimensiones que determinan la percepción que tienen los pacientes sobre el servicio prestado en este centro clínico, con la finalidad de que el mismo plantee las necesidades que se presentan y se tomen acciones al respecto. Los servicios de salud son aquellos prestados por los médicos, odontólogos, enfermeras, y el resto del personal de hospitales, consultorios y clínicas, con el propósito de conservar o restablecer la salud (Losada y Rodríguez, 2007).

La evaluación realizada por los pacientes de la calidad del servicio que han recibido es importante para introducir estrategias de mejora en la calidad asistencial (Regaira, et al., 2010); de aquí la necesidad de realizar esta investigación en el caso de estudio de un centro médico privado.

Este artículo se estructura de la siguiente manera: primero se presenta, a manera de revisión de literatura y como parte de la introducción previa, una sección de aspectos importantes de la calidad de servicios en el área de salud, la cual permitirá contextualizar al lector en el tema abordado. En la siguiente sección, se describe la metodología usada, basada en la aplicación de una versión de la encuesta SERVQUAL, el Análisis de Factores para identificar las dimensiones, y la determinación mediante métodos estadísticos de la fiabilidad y validez del instrumento usado. Finalmente, se presentan los resultados y el análisis de los mismos, lo cual sustenta las conclusiones presentadas.

\section{CALIDAD DE SERVICIOS EN EL ÁREA DE SALUD}

Aun cuando la calidad en los servicios de salud ha sido un tema ampliamente tratado en la literatura desde hace más de 40 años, su definición, interpretación y evaluación continúa siendo polémica y compleja, debido a los múltiples factores involucrados en su constructo (Cabello y Chirinos, 2012). Para los años 90, la calidad de servicio percibida por el cliente era una evaluación poco frecuente en el sector sanitario dónde se prefería medir la satisfacción del paciente respecto a una interacción puntual con un profesional, dispositivo o servicio del sistema sanitario (Mira, Buil, Rodríguez y Aranaz, 1997).

Según Losada y Rodríguez (2007), la conceptualización y medición de la calidad de servicio en salud se ha abordado, por lo menos, desde dos enfoques. Uno que nace en los años 80 's con los trabajos de Donabedian (1980, 1988, citados por Losada y Rodríguez, 2007), quien define la calidad de los servicios de salud como la habilidad de alcanzar objetivos deseables haciendo uso de medios legítimos. El otro enfoque al que hacen mención, es el de quienes sostienen que la percepción de un paciente sobre la prestación de un servicio, determina, en forma definitiva, su nivel de calidad.

Realizando una revisión general de la literatura en investigaciones en el sector de salud, se destacan los trabajos de quienes validan una versión del SERVQUAL en el hospital (SERVQHOS) (Mira et al., 1997); Mira, Aranaz, Lorenzo, Rodríguez y Moyano (2001) quienes usando el SERVQHOS, comparan los resultados de dos hospitales públicos; Díaz (2005) por su parte, analiza la calidad del servicio sanitario público desde la perspectiva del cliente, tomando como base el modelo SERVPERF e identifica las dimensiones que conforman la calidad de dicho servicio a través de un Análisis de Factores; Regaira et al (2010) quienes evalúan la calidad asistencial en cuidados intensivos a partir de la precepción de los pacientes mediante la escala SERVQUAL; Jélvez, Riquelme y Gómez (2010) evalúan la calidad del servicio médico ofrecido a sus pacientes por el Centro de Salud 
Familiar en Chile utilizando el modelo SERVQUAL; Cabello y Chirinos (2012) quienes validan y evalúan la aplicabilidad de encuestas para medir la satisfacción de los usuarios en consulta externa y emergencia de un hospital público en Perú; entre muchos otros.

\section{METODOLOGÍA}

El desarrollo de ésta investigación se realizó en un centro de salud privado ubicado en la ciudad de Valencia (Venezuela), en el cual se llevó a cabo una investigación de tipo cuantitativa, con la ayuda de herramientas del campo estadístico, pero basada en una investigación documental que permitió sentar las bases teóricas del tema abordado, a manera de estado del arte.

Así mismo dentro de la investigación cuantitativa éste trabajo se centra en la investigación descriptiva porque busca explicar las dimensiones que determinan el nivel de satisfacción de los usuarios de un centro médico privado a través del análisis estadístico (Rojas, 2001).

La técnica utilizada para la recolección de datos fue la encuesta, en la cual se incluye la Escala de veintidós (22) ítems destinados a la determinación de las percepciones de la calidad de servicio y cinco (5) ítems que miden la percepción en general de la calidad de servicio en el centro médico privado entre otros aspectos, y que serían utilizadas para análisis de validez de la escala usada.

La encuesta presentaba una escala de respuesta tipo Lickert, con amplitud 7, donde el menor valor posible es 1 , cuyo significado es que el usuario del centro médico asistencial privado está en total desacuerdo y la calificación más alta es 7 , la cual indica que el encuestado está totalmente de acuerdo.

Las variables de la encuesta están diseñadas para medir las dimensiones de calidad de servicio, con base en las percepciones que plantean Parasuraman et al. $(1998,1991)$. Estas dimensiones teóricas son las siguientes:

Empatía: atención personalizada, horarios accesibles, instalaciones atractivas al usuario.

Confiabilidad: habilidad del personal de brindar al usuario el servicio tal como se ofreció.

Seguridad: actitud del personal para transmitir confianza y credibilidad al usuario.

Capacidad de respuesta: disponibilidad del personal a ayudar al usuario, responder sus dudas, brindarle el tiempo necesario.
Tangibilidad: apariencia de las instalaciones físicas, del personal y de los equipos utilizados.

La población estuvo constituida por todos los usuarios del centro médico privado que hicieron uso de éste servicio durante el estudio. Para dicha investigación la muestra constó de cien (100) personas elegidas mediante un muestreo por conveniencia del investigador, clasificado dentro del tipo de muestreo no probabilístico. Vale la pena señalar que en este contexto de salud, es determinante la disposición del paciente para colaborar en el estudio, por lo que resulta inadecuado el uso de muestreo probabilístico.

El instrumento de medida debe cumplir con las propiedades de fiabilidad, validez y poder de diagnóstico (Molina, 2008), por ello a los resultados obtenidos de la aplicación de la encuesta se les realizó un análisis descriptivo de todas las variables haciendo uso del paquete estadístico SPSS $®$, a través del cual se analizó la consistencia del cuestionario para determinar la fiabilidad del mismo, haciendo el cálculo del coeficiente Alfa de Cronbach.

Para determinar la relación que existe entre las variables, condición inicial para el uso adecuado de la técnica estadística de Análisis de Factores, se procedió a calcular el determinante de la matriz de correlaciones y el índice $\mathrm{KMO}$, indicadores usados para demostrar la adecuación de la muestra para la aplicación de la técnica estadística multivariante de Análisis de Factores.

El determinante de la matriz es un indicador del grado de intercorrelación, si es muy pequeño, es decir cercano a cero (0), indica que el grado de intercorrelación es muy grande, condición inicial que debe cumplir el análisis (Pérez, 2005; Vicente y Oliva y Manera, 2003).

Por su parte, el KMO (Kaiser-Meyer-Olkin) es una medida basada en los coeficientes de correlación observados de cada par de variables y en sus coeficientes de correlación parcial. En el caso de que exista adecuación de los datos a un modelo factorial, el KMO será próximo a la unidad (1); mientras más cerca esté de 1 , mayor es la adecuación de los datos, considerándose valores aceptables los mayores a 0,5, y excelentes los mayores a 0,9 (Pérez, 2007).

Por último se realizó un análisis de la validez del cuestionario basado en pruebas estadísticas. Las encuestas que evalúan percepciones requieren de una serie análisis para evidenciar sus propiedades psicométricas que garanticen su fiabilidad y validez, por lo tanto, es necesario contar con encuestas 
validadas, confiables y de fácil aplicación para medir la calidad de servicio e identificar los aspectos clave que determinan la satisfacción de los usuarios externos en servicios de salud y que permitan implementar acciones de mejora (Cabello y Chirinos, 2012).

\section{RESULTADOS Y ANÁLISIS}

Para procesar los datos de una manera eficiente e identificar las dimensiones que influyen en la calidad de servicio del centro de salud privado en estudio, se utilizó el método de Análisis de Factores, modelo estadístico multivariado de reducción de datos que proporciona correlación de las variables y además permite reorganizar o reducir las variables a ciertos valores que serán sometidos a análisis posteriores (Namakforoosh, 2000).

Se contó con la ayuda del software SPSS para realizar el Análisis de Factores, en el cual primeramente se debe introducir los datos obtenidos mediante la encuesta realizada; dicha encuesta, como ya se ha señalado, contó con veintidós (22) preguntas en las cuales se reflejaban las cinco (5) principales dimensiones de la calidad de servicio como lo son, la tangibilidad, la seguridad, la empatía, la capacidad de respuesta y la confiabilidad.

El primer resultado de interés al momento del Análisis de Factores es el referente al determinante de la matriz de correlaciones, ya que este indica el grado de correlación existente entre las variables, si este determínate arroja un valor muy cercano a cero indicaría que las variables se encuentran linealmente relacionadas y es válido el uso del análisis, de lo contrario no se justifica el uso.

En el caso de este estudio el determinante de la matriz de correlaciones con veintidós (22) variables arrojó como valor $1,01 \times 10-8$, lo cual da una evidencia de lo adecuado del estudio.

Seguidamente se estimó la confiabilidad de toda la escala mediante el Alfa de Cronbach, que es el coeficiente que determina la consistencia interna de una escala de medida analizando la correlación de media de una variable con todas las demás que integran dicha escala (Gonzales 2009), este arrojó 0,955 para toda la escala considerándose muy bueno ya que debe estar entre 0,8 y 1 para decir que la consistencia es significativa.

Aun cuando en esta etapa no se evalúa la fiabilidad de la escala como tal, el coeficiente Alfa de Cronbach, por basarse en correlaciones entre las variables, da una idea de lo adecuado del empleo del Análisis de Factores. Así, posteriormente al analizar la relación existente de cada variable con el Alfa de Cronbach de toda la escala se observa que ninguna variable genera un cambio significativo en el coeficiente global.

Para la extracción de los factores se seleccionó la técnica de Componentes Principales. Ésta técnica estadística que permite transformar un conjunto de variables interrelacionadas en otro conjunto de variables no correlacionadas denominadas factores, siendo estos factores combinación lineal de las variables originales (Quintín y Santana 2007).

Los criterios utilizados para obtener el número de factores se basaron en que los auto valores fueran mayores a uno (1) y que la varianza explicada fuese al menos $50 \%$ del total, como resultado se obtuvo una varianza total explicada de $66,631 \%$ lo cual satisface la condición inicial.

Una vez realizada la extracción hace la rotación de los factores con el fin de facilitar su interpretación; para esto se utilizó el método VARAMAX, el cual reduce el número de variables con saturación elevada en un mismo factor, lo que mejora su interpretación (Pardo y Ruiz, 2005).

\subsection{Identificación de dimensiones}

Realizada la rotación se identificaron tres (3) dimensiones con las veintidós (22) variables las cuales fueron reducidas a veinte (20) buscando la forma más sencilla e interpretable. Esta estructura rotada es presentada en la Tabla 1.

En el primer factor, se agrupan variables que tienen que ver con la habilidad del personal del centro médico para inspirar confianza, destacándose el comportamiento del personal y su amabilidad; en este factor se agrupan variables que Parasuraman et al. (1988, 1991), incluían en Seguridad.

En el segundo factor, identificado como confiabilidad, se destacan aquellas variables relacionadas con la habilidad para realizar el servicio prometido de forma fiable y cuidadosa; la paciencia, el cumplimiento del servicio en el tiempo prometido y la disponibilidad, se incluyen en este factor.

En el tercer factor, planteado también por Parasuraman et al (ob. cit.) en su SERVQUAL, se destaca la Tangibilidad o Elementos tangibles, donde se incluye la apariencia de las instalaciones y equipos. 
Tabla 1. Matrix de componentes rotados

\begin{tabular}{|l|r|r|r|}
\hline \multirow{2}{*}{} & \multicolumn{3}{|c|}{ Componentes Rotados } \\
\cline { 2 - 4 } & $\mathbf{1}$ & $\mathbf{2}$ & \multicolumn{1}{c|}{$\mathbf{3}$} \\
\hline VAR15 &, 786 & & \\
\hline VAR16 &, 783 & & \\
\hline VAR17 &, 743 & & \\
\hline VAR19 &, 736 & & \\
\hline VAR18 &, 727 & & \\
\hline VAR14 &, 727 & & \\
\hline VAR20 &, 659 & & \\
\hline VAR13 &, 611 & & \\
\hline VAR08 &, 584 & & \\
\hline VAR06 & &, 792 & \\
\hline VAR03 & &, 780 & \\
\hline VAR10 & &, 745 & \\
\hline VAR12 & &, 684 & \\
\hline VAR05 & &, 644 & \\
\hline VAR07 & &, 547 & \\
\hline VAR01 & & &, 818 \\
\hline VAR22 & & &, 692 \\
\hline VAR04 & & &, 658 \\
\hline VAR09 & & &, 607 \\
\hline VAR02 & & &, 531 \\
\hline
\end{tabular}

Fuente: Elaboración propia

Tabla 2. Dimensiones de la Calidad de Servicio del caso de estudio

1: Seguridad. Atención y habilidad del personal
para inspirar confianza
V17 El comportamiento del personal del centro le
inspira confianza
V16 Las personas que trabajan en la centro son
amables con usted
V19 El personal realiza bien el servicio desde la
primera vez
V18 El personal del centro le presta atención única
cuando la necesita
V15 Usted siente confianza en el médico que le atendió.
V13 El servicio en el centro médico es puntual
V14 Confía usted en los equipos médicos utilizados en
el centro médico
V20 Considera usted que los horarios de atención del
centro son accesibles
V08 La apariencia (limpieza y uniforme) del personal
es pulcro.

Dimensión 2: Confiabilidad. Habilidad para realizar el servicio prometido de forma fiable y cuidadosa

V03 El personal lo trato con amabilidad, respeto y paciencia.

V06 Cuando el personal del centro le promete hacer algo en cierto tiempo, lo hace

V10 La disponibilidad del personal para ayudarle cuando lo necesita fue satisfactoria.

V12 El médico le brindo el tiempo necesario para contestar sus dudas.

V05 El médico le explicó a usted o a sus familiares con palabras fáciles de entender el resultado del procedimiento o análisis que le realizaron.

V07 El personal de enfermería muestra interés por los pacientes.

Dimensión 3: Tangibilidad. Apariencia de las Instalaciones físicas y equipos

V01 Las instalaciones físicas del servicio tienen apariencia moderna.

V04 Los consultorios contaron con los equipos y materiales necesarios para su atención.

V09 El consultorio y sala de espera estuvieron limpios, cómodos y acogedores.

V22 Las instalaciones del centro son visualmente atractivas

V02 Su atención se realizó respetando el orden de llegada.

Fuente: Elaboración propia.

La capacidad de respuesta y la empatía, los otros factores del modelo SERVQUAL, a pesar de no identificarse dentro de la estructura subyacente de los datos generados en este estudio, se ven reflejadas en los tres con los que se ha identificado el modelo en cuestión; así, la puntualidad, atención individualizada, son reflejadas en éste.

\subsection{Validez de la Encuesta}

De acuerdo con lo planteado por Hernández, Fernández y Baptista (2010), la validez de una escala se refiere al grado en que dicha escala realmente mide la variable que pretende medir.

Para determinar la validez de la encuesta aplicada, se usan los tres enfoques clásicos: validez de Contenido, validez de Constructo y validez de Criterio.

La validez de Contenido se refiere al grado en que un instrumento refleja el dominio especifico de contenido de lo que se mide (Hernández et al., 2010). 
Las veintidós variables que integran la escala son una adaptación del conocido modelo SERVQUAL. EI modelo SERVQUAL (Parasuraman et al., 1988, 1994) es el más usado en la actualidad para medir la calidad de servicio; en lo que respecta a su aplicación en el sector salud, es mucha la evidencia presentada en la literatura del tema (Mira, Aranaz, Lorenzo, Rodríguez y Moyano, 2001; Díaz, 2005; barrios y Mejías, 2008; Regaira et al., 2010; Jélvez, Riquelme y Gómez, 2010; Cabello y Chirinos, 2012; entre otros), por lo que se puede considerar que ésta encuesta presenta validez de contenido.

La validez de criterio o validez pragmática, establece la validez de un instrumento de medición comparándola con algún criterio externo (Hernández et al, 2010); si se fija un criterio en el presente se conoce como Concurrente, y si se fija el criterio en el futuro, se le denomina Predictiva.

En los casos en estudio, para determinar la validez concurrente, se clasificaron los encuestados en dos categorías, una formada por puntuaciones menores a la media y otros con puntuaciones mayores, con baja percepción y con alta percepción respectivamente, siguiendo la metodología planteada por Mejías y Maneiro (2007). Tomando como referencia una pregunta adicional presentada en el instrumento relacionada con la percepción general de la calidad de servicio ofrecida, y con base en una prueba estadística no paramétrica ( $U$ de Mann-Whitney), se encontró que existen diferencias significativas (al $5 \%$ ) entre las medias de las categorías, lo cual evidencia la validez concurrente de la encuesta. Para evidenciar la validez predictiva, se realizó un análisis de regresión tomando como variables independientes los valores promedios de las dimensiones $(\mathrm{Xi})$ y como variable dependiente (Y) una variable adicional a la escala que media la satisfacción de los usuarios sobre los servicios académicos prestados. Los resultados estadísticos (R2=0,526 y pruebas de adecuación del modelo significativos al 5\%) evidencian la validez predictiva del modelo.

La validez de Constructo o validez de concepto, se refiere al grado en el que una medición se relaciona de manera consistente con otras mediciones, de acuerdo con hipótesis derivadas teóricamente y que conciernen a los conceptos que se están midiendo (Hernández at al., 2010), se puede clasificar en convergente y discriminante.

Para evidenciar la validez convergente, se realizó un análisis de correlación con los valores promedios de la Encuesta y una variable adicional presentada a los encuestados que medía la Calidad de Servicios en general. Los resultados (pruebas significativas la $5 \%$ ) confirman la validez convergente de la escala para el caso en estudio.

\subsection{Análisis de Fiabilidad}

fiabilidad que no es más que el grado en el que un instrumento mide con precisión y sin error (Izquierdo 2008). Existen muchos enfoques para este fin; en este estudio de caso se uso el coeficiente de consistencia interna Alfa de Cronbach.

Los valores alcanzados para el coeficiente Alfa de Cronbach en los casos presentados fueron superiores a $0,800(0,830,0,878,0,930$, para cada dimensión, respectivamente), lo cual indica una homogeneidad y equivalencia de respuesta a todos los ítems a la vez y para todos los encuestados.

La alta consistencia interna es un indicador suficientemente válido de que la escala usada mide la Calidad de Servicio en el caso estudiado, lo que se interpreta como garantía de una alta fiabilidad de la escala.

\section{CONCLUSIONES}

La investigación acerca de la calidad de servicio de un centro hospitalario privado se llevó a cabo primeramente mediante una fase con un enfoque teórico y luego una fase que se basó en la medición y en el análisis estadístico.

El instrumento de medición utilizado fue aplicado a una población de cien (100) personas que visitan frecuentemente el centro hospitalario, este instrumento conto con cinco (5) dimensiones y veintidós (22) variables, presentando una validez tanto predictiva como de contenido.

Realizado el análisis estadístico mediante el programa IBM SPSS $®$ se pudo notar que las dimensiones que realmente influye en la calidad de servicio son tres (3), a saber: Seguridad, Confiabilidad y Tangibilidad; y que las variables solo veinte determinan la calidad de los servicios en el caso de estudio.

Al determinar estas dimensiones que influyen en la calidad de servicio del centro médico privado se pueden realizar estudios posteriores que permitan generar análisis estratégicos para buscar mejoras de la calidad de servicio, así como también analizar donde se encuentran las fallas y cuáles podrían ser las futuras amenazas.

Estos resultados servirían para generar planes para fortalecer estas dimensiones y mantener el 
alto nivel de la calidad de servicio que actualmente se presta el cual fue arrojado por el estudio.

Nota: Este artículo forma parte de los resultados del proyecto de investigación de la Cátedra de Gestión de la Calidad de la Escuela de Ingeniería Industrial de la Universidad de Carabobo. Fue presentado en el marco de las "XI Jornadas de Investigación 2013: Ingeniería Aplicada" de la Universidad Nacional Experimental Politécnica "Antonio José de Sucre", Vice-rectorado Puerto Ordaz, Venezuela.

\section{REFERENCIAS BIBLIOGRÁFICAS}

[1] Barrios, M. y Mejías, A. (2008). Dimensiones de la Calidad de Servicio en un Centro Médico Asistencial Universitario. Revista Ingeniería Industrial: Actualidad y Nuevas Tendencias, 1 (1), 21-30.

[2] Cabello, E. y Chirinos, J. (2012).Validación y aplicabilidad de encuestas SERVQUAL modificadas para medir la satisfacción de usuarios externos en servicios de salud. Rev Med Hered, 23(2), 88-95.

[3] Deming, W (1986). Calidad, Productividad y Competitividad (Edición en Español. Madrid: Laves.

[4] Díaz, (2005). Dimensiones de la calidad del servicio sanitario. Especial referencia a la Sanidad Pública. ESIC MARKET, 5, 371-398.

[5] Izquierdo. M (2008), Biomecánica y Bases Neuromusculares de la Actividad Física y el Deporte. Medica panamericana.

[6] Guillermo, E., y Casalino, C. (2008). Calidad de servicio de la consulta externa de Medicina Interna de un hospital general de Lima mediante la encuesta Servqual. Revista Sociedad Peruana de Medicina Interna, 21 (2008), N4, 145-146.

[7] Guillo, J (2000). Calidad total: Fuente de ventaja competitiva. Publicaciones Universidad de Alicante.

[8] González. P (2009). Manual de medicina de urgencia y emergencia. Universidad de Oviedo.

[9] Hernández, R., Fernández, C. y Baptista, L. (2010). Metodología de la Investigación. México: McGrawhill.

[10] Jélvez, A.; Riquelme, Y. y Gómez, N. (2010). Evaluación de la calidad de servicio en centro de salud familiar en Chile. Horizontes Empresariales, 9 (2), 51-66.
[11] Losada, M. y Rodríguez, A. (2007). Calidad de Servicio de Salud: una revisión a la literatura desde la perspectiva del marketing. Cuadernos de Administración, 20 (34), 237-258.

[12] Mejías, A. y Maneiro, N. (2010). Medición de la calidad de servicios. Serie de Cuadernos de Ingeniería industrial. Venezuela: Universidad de Carabobo.

[13] Mira, J.J., Buil, J.A., Rodriguez-Marín, J. y Aranaz, J. (1997). Calidad percibida del cuidado hospitalario. Gaceta Sanitaria, 11: 176-189.

[14] Mira, J., Aranaz, J., Rodríguez, J., Buil, J., Castell, M., Vitaller, J. (1998). SERVQHOS: un cuestionario para evaluar la calidad percibida de la asistencia hospitalaria. Medicina Preventiva, 4, 12-18

[15] Mira, J.; Aranaz, J.;Lorenzo, S.; Rodríguez, J. y Moyano, S. (2001). Evolución de la Calidad Percibida por los pacientes en dos hospitales públicos. Psicothema, 13 (4), 581-585.

[16] Molina, X. (2008). La estructura y naturaleza del capital social en las aglomeraciones territoriales de empresas: Una aplicación al sector cerámico español. Fundación BBVA.

[17] Namakforoosh. M (2000), Metodología de la Investigación. Limusa.

[18] Norma Internacional ISO 9000 (2005). Sistemas de Gestión de la Calidad. Términos y Definiciones. Ginebra, International Organization for Standardization.

[19] Pardo, A. y Ruiz, M. 2005. Análisis de datos con SPSS. Madrid: McGraw-Hill/Interamericana de España.

[20] Parasuraman, A.; Zeithaml, V. y Berry, L. (1988). SERVQUAL: A Multiple-Item Scale for Measuring Customer Perceptions of Service Quality, Journal of Retailing, 12-40.

[21] Parasuraman, A.; Zeithaml, V. y Berry, L. (1994). Reassessment of Expectations as a Comparison Standard in Measuring Service Quality: Implications for Future Research, Journal of Marketing, 1, 11-24.

[22] Pérez, C. (2005). Métodos Estadísticos Avanzados con SPSS. Madrid: Thomson.

[23] Rojas, E. (2011). Metodología de la Investigación. Recuperado el 20 de Febrero de 2013, de http://metodologiaeconomia2011. blogspot.com/2011/05/investigacioncuantitativa.html 
[24] Quintín, M. y Santana, Y. (2007). Tratamiento estadístico de datos SPSS. Paraninfo.

[25] Regaira, E.; Sola, M.; Goñi, R.; Del Barrio, M.; Margall, M.; Asiain, M. (2010). La calidad asistencial en cuidados intensivos evaluada por pacientes mediante la escala SERVQUAL. Enfermería Intensiva, 21 (1), 3-10.
[26] Zeithaml, V., Parasuraman, A. y Berry, L. (1992). Calidad total en la Gestión de Servicios. Madrid: Diaz de Santos.

[27] Vicente y Oliva, M. de y Manera, J. (2003). El análisis factorial y por componentes principales. En Lévy, J. y Valera, J. (Comp.), Análisis Multivariable para las Ciencias Sociales. Madrid: Pearson Educación. 